\title{
Breakdown of the mean field for dark solitons of dipolar bosons in a one-dimensional harmonic trap
}

\author{
Michał Kowalski $\odot,{ }^{1,2, *}$ Rafał Ołdziejewski $\odot,{ }^{1}$ and Kazimierz Rzazżewski ${ }^{1}$ \\ ${ }^{1}$ Center for Theoretical Physics, Polish Academy of Sciences, Al. Lotników 32/46, 02-668 Warsaw, Poland \\ ${ }^{2}$ Faculty of Technical Physics, Poznan University of Technology, Piotrowo 3, 60-965 Poznan, Poland
}

(Received 29 November 2019; accepted 15 April 2020; published 23 June 2020)

\begin{abstract}
We directly compare the mean-field and the many-body approaches, in a one-dimensional Bose system in a harmonic trap. Both contact and dipolar interactions are considered. We obtain eigenstates of the many-body system by exact diagonalization with a truncated basis. We propose a multiatom version of the phase-imprinting method to generate dark solitons in the system. We begin with a general analysis of system dynamics and observe the emergence of a dark soliton and a shock wave in the form of sloshing motion of the condensate upon phase imprinting. The center of mass and soliton go out of phase because the soliton does not oscillate with the trap frequency. A detailed investigation of frequencies reveals significant differences in the results obtained in the mean-field versus the many-body picture.
\end{abstract}

DOI: 10.1103/PhysRevResearch.2.023386

\section{INTRODUCTION}

Solitons-solutions of nonlinear integrable differential equations which propagate without dispersion-appear across many areas of science, ranging from physics to biology and medicine [1]. Among a number of known equations supporting solitonic solutions an important example constitutes the nonlinear Schrodinger equation, also called the Gross-Pitaevski equation (GPE). Although mainly used in the context of weakly (characterized by negligible ground-state depletion) interacting ultracold bosons [2], it also describes the properties of the electric field of light in nonlinear media [3].

If atoms in a Bose-Einstein condensate (BEC) repel each other by pointlike interactions, a resulting solitonic solution of the GPE takes the form of a dark soliton: a density dip with a phase jump across its density minimum. An analytical expression describing dark solitons in a uniform gas characterized by the GPE was found in the 1970s [4-7]. Shortly after achievement of the first condensation in 1995, dark solitons were successfully generated by the phase-imprinting method in experimental setups with ultracold ${ }^{87} \mathrm{Rb}$ [8] and ${ }^{23} \mathrm{Na}$ [9] atoms trapped in a harmonic confinement.

One of the most classic results regarding dark solitons in a BEC interacting only by short-range forces concerns its dynamics in a harmonic trap. In the so-called Thomas-Fermi (TF) regime for ultracold bosons, the characteristic frequency of the soliton's oscillations is expressed by the trapping frequency $\omega$ and equals $\omega / \sqrt{2}$ [10], which was also observed in the experiment [11]. Studying the dynamics of trapped

\footnotetext{
"michal.ry.kowalski@student.put.poznan.pl

Published by the American Physical Society under the terms of the Creative Commons Attribution 4.0 International license. Further distribution of this work must maintain attribution to the author(s) and the published article's title, journal citation, and DOI.
}

solitons for contact interactions remains an active field of research, with examples exploring different physical scenarios given in Refs. [12-14]. However, the remarkably robust result obtained for short-range interactions in the TF regime does not hold for dipole-dipole atomic interactions [15]. In this case, the oscillation frequency of solitonic structures in a repulsive dipolar BEC - predicted only in 2015 [16,17]depends greatly on the strength of the atomic interactions and the interplay between local and nonlocal contributions to the total energy. With the recent progress on quantum gases consisting of atoms with considerable magnetic moments like ${ }^{164}$ Dy $[18,19]$ and Er [20], dipolar systems are now within experimental reach and call for deeper analysis.

The nonlinear mean-field (MF) theory of ultracold bosons provides an approximate description of interacting cold atoms. The underlying many-body (MB) model is linear and the state of the system is given by the MB wave function depending on the positions of all particles. The discussion of correspondence between dark solitons present in the MF and MB solutions of the full Hamiltonian has a long history. The best-known example refers to the link between dark solitons moving on the circumference of a ring and type II excitations from the seminal Lieb-Liniger model $[21,22]$ in the context of contact interacting particles (see [23-33] and references therein). However, little is known about MB states possessing features of dark solitons in a one-dimensional (1D) harmonic trap for both contact and purely dipolar interactions. Here, we aim to partially bridge this gap by studying many-body dark solitons in weakly interacting trapped systems of only a few atoms far beyond the TF regime. In particular, we investigate the oscillations of the $\mathrm{MB}$ solitons and compare them to the dark solitons described by the GPE. Our results not only establish a link between the MF approach and the full many-body theory, but also can be verified in modern experiments with precise control over only a few atoms in optical lattices or single traps (see, for instance, [34-38]).

The work is organized as follows. In Sec. II, we introduce our many-body model for atoms interacting repulsively by 
contact or purely dipolar forces in a quasi-1D harmonic trap. We also recall the corresponding GPE. Then, in Sec. III, we analyze the many-body eigenstates of the system. Following previous papers regarding MB solitons in the Lieb-Liniger model, we expected the eigenstate to form a dip in density and a phase jump. Surprisingly, due to the peculiar properties of the harmonic trap, there is no good candidate for a many-body solitonic state among the eigenstates. Therefore, we introduce the many-body phase-imprinting method of creating dark solitons in a harmonic trap. In Sec. IV, we finally present our results. We investigate dynamics of the MB solitons. We calculate the oscillation frequencies for different coupling strengths for both types of interactions and compare our findings with the GPE. The most important result is the significant disagreement in frequencies obtained in the MB versus the MF approach.

\section{THE MODEL}

We investigate a system of $N$ repulsive bosons trapped in a harmonic potential,

$$
U(x, y, z)=\frac{1}{2} m \omega^{2} x^{2}+\frac{1}{2} m \omega_{\perp}^{2}\left(y^{2}+z^{2}\right) .
$$

We assume that the transverse confinement is tight, so the wave function stays in the lowest energy level in the $Y$ and $Z$ directions, meaning that our system is quasi-1D. The aim of our study is to compare contact and dipolar solitons in the many-body and mean-field approaches. The system of $N$ repulsive bosons is often approximated using the quasi-1D GPE,

$$
\begin{aligned}
i \frac{\partial \psi_{\mathrm{GPE}}(\mathbf{r}, t)}{\partial t}= & \psi_{\mathrm{GPE}}(\mathbf{r}, t)\left(-\frac{1}{2} \frac{\partial^{2}}{\partial x^{2}}+U(\mathbf{r})+(N-1)\right. \\
& \left.\times \int d \mathbf{r}^{\prime}\left|\psi_{\mathrm{GPE}}(\mathbf{r}, t)\right|^{2} V\left(\mathbf{r}-\mathbf{r}^{\prime}\right)\right), \quad(2 \mathrm{a})
\end{aligned}
$$

$\int d \mathbf{r}\left|\psi_{\mathrm{GPE}}(\mathbf{r}, t)\right|^{2}=1$,

where $U(\mathbf{r})$ is the trapping potential and $V(\mathbf{r})$ is an interaction potential. Throughout the paper we use oscillatory units with $\hbar \omega, \sqrt{\frac{\hbar}{m \omega}}, \sqrt{\hbar m \omega}$, and $\frac{1}{\omega}$ as units of energy, length, momentum, and time, respectively. In the context of ultracold atoms, this equation is also called the MF description of weakly interacting bosons. This approach provided correct predictions of many properties of the BEC, including its shape, energy, and normal modes of excitations and many other nonlinear phenomena. Moreover, the GPE supports dark solitons in the form of solutions with a density notch and a quickly changing phase, which have also been experimentally produced with the phase imprinting method. However, the MF model assures a simplified description of a system of $N$ repulsive bosons based on the naive assumption that every atom is in the same state. It is only an approximation of the more fundamental many-body approach. As the nonlinear GPE supports solitons, it is important to look for solitons in the linear MB approach and compare them. This has been done in the Lieb-Liniger model [21,22], but to the best of our knowledge, this is the first paper in which multiatomic solitons are considered for the harmonically trapped system.
In order to describe a system of $N$ bosons following the many-body approach, one needs to derive the wave function depending on the positions of all particles. One possible way of deriving the $\mathrm{MB}$ wave function is to diagonalize a Hamiltonian matrix. The Hamiltonian of the system under investigation can be written in the form

$$
H=\sum_{i}^{N} T_{i}+\sum_{i}^{N} \frac{1}{2} x_{i}^{2}+\sum_{i<j}^{N} V_{i j}
$$

with $T_{i}$ being the single-particle kinetic energy operator and $V_{i j}$ the two-body interaction operator. Hamiltonian (3) can be rewritten as the sum of the Hamiltonian of the noninteracting quantum harmonic oscillator and the interaction term. Therefore, in the second quantization Hamiltonian (3) reads

$H=H_{\mathrm{osc}}+\frac{1}{2} \int \hat{\Psi}^{\dagger}(x) \hat{\Psi}^{\dagger}(y) V(x-y) \hat{\Psi}(x) \hat{\Psi}(y) d x d y$,

with $\hat{\Psi}(x)$ being a bosonic field operator and $H_{\mathrm{osc}}=$ $\frac{1}{2} \int \hat{\Psi}^{\dagger}(x)\left(-\frac{\partial^{2}}{\partial x^{2}}+x^{2}\right) \hat{\Psi}(x) d x$. We study systems where $V(r)$ is either a short-range $V_{\mathrm{sr}}(r)$ or a long-range dipolar $V_{\mathrm{dd}}(r)$ potential. The short-range potential is $V_{\mathrm{sr}}(r)=g_{\mathrm{sr}} \delta(r)$, with the parameter $g_{\mathrm{sr}}=\int V_{\mathrm{sr}}(r) d r$ defining the strength of the interaction. We study repulsive systems with $g>0$. In order to obtain the explicit formula describing $V_{\mathrm{dd}}$, we follow the procedure of dimensional reduction described in Refs. $[15,16]$.

We introduce the aspect ratio of the trap $\sigma=\frac{\omega_{\perp}}{\omega}$ and the dipolar coupling strength $A_{\mathrm{dd}}$, yielding

$$
V_{\mathrm{dd}}(r)=\frac{A_{\mathrm{dd}}}{\sigma^{2}} \frac{1}{\sigma} V_{\mathrm{eff}}\left(\frac{r}{\sigma}\right),
$$

with the term

$$
V_{\mathrm{eff}}(u)=\frac{3}{4}\left[-2|u|+\sqrt{2 \pi}\left(1+u^{2}\right) e^{u^{2} / 2} \operatorname{Erfc}\left(\frac{|u|}{\sqrt{2}}\right)\right] .
$$

This effective quasi-1D potential comes from the integration of the full 3D dipolar interaction over both transverse variables. In fact, the reduction of the 3D dipole-dipole potential to quasi-1D geometry produces two terms. One of them, $V_{\mathrm{dd}}(r)$, is continuous and finite at zero distance between the atoms. The other one is a delta function. As the area of our interest is the long-range part of the interaction, we assume that the contact term of the effective dipolar potential is exactly canceled, possibly with the help of Feshbach resonances. The shape of the potential is shown in Fig. 1.

As we want to investigate similarities and differences between systems interacting via contact and dipolar forces, we define the dipolar strength parameter $g_{\mathrm{dd}}$ :

$$
g_{\mathrm{dd}}=\int V_{\mathrm{dd}}(r) d r=\frac{3 A_{\mathrm{dd}}}{\sigma^{2}} .
$$

Henceforth, we keep $\sigma=0.1$ and compare systems described by the same strength parameters $g_{\mathrm{dd}}=g_{\mathrm{sr}}=g$. It is important to mention that in the case of the MF approach only a gas parameter $(N-1) g$ defines the system. However, in the multiatom approach both $N$ and $g$ are separately relevant.

As we are investigating a weakly interacting system we chose the quantum harmonic oscillator eigenstates as the basis. We use the second quantization formalism and define a basis of Fock states. We take into account all states with 


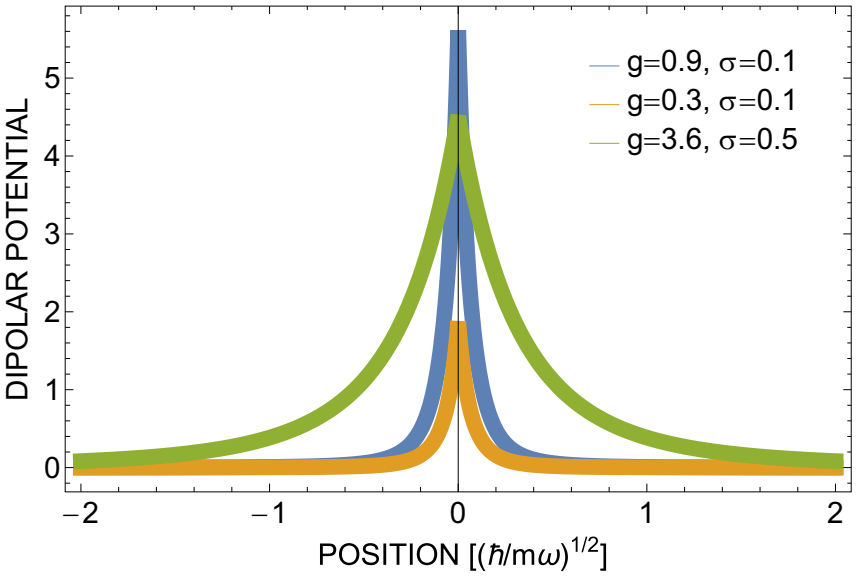

FIG. 1. Repulsive dipolar potential for a variety of parameters $\sigma$ and $g$. Throughout this paper we choose to keep $\sigma=0.1$ and modify the strength of the interaction by varying the parameter $g$.

a given number of particles whose energies are lower than a cutoff energy. Defining the cutoff in terms of the total energy rather than the energies of individual atoms significantly reduces the dimensionality of the relevant Hilbert subspace [39].

In order to obtain eigenstates and energies of the system, we diagonalize the Hamiltonian matrix

$$
H_{i j}=\langle i|\hat{H}| j\rangle,
$$

where $|i\rangle$ and $|j\rangle$ are states belonging to the Fock space.

\section{SOLUTIONS}

Having access to both eigenenergies and eigenstates we are ready to look for solitons in the system. Following recent papers investigating many-body solitons in the Lieb-Liniger model $[29,40]$, one might think that also in this case dark solitons could be identified among eigenstates of Hamiltonian (4).

Studying the repulsive case, we are interested in the properties of dark solitons. In this situation the density forms a single notch and, in the area of the notch phase, exhibits a jump of $\pi$. Keeping this in mind, one can ask the question whether any single-particle state fulfills these conditions.

We start our analysis with the ideal noninteracting gas. In this case, the excited state of the quantum harmonic oscillator, $\bigotimes^{N}|0,1,0, \ldots\rangle=|0, N, 0, \ldots\rangle$, seems like a reasonable candidate because it has both the density dip and the phase jump, so one can try to find the eigenstate of the interacting system with the highest contribution of the aforementioned state among all the Fock states.

This turns out to be nontrivial even for weak interactions. We expected the maximum $|0, N, 0, \ldots\rangle$ occupation to tend to 1 as the interactions become weaker. Instead, it approached different values depending on the number of particles considered. This is a peculiar property of the harmonic trap caused by evenly spaced energies of $H_{\mathrm{osc}}$. Once the interactions become weaker, the energy of the eigenstate with the highest $|0, N, 0, \ldots\rangle$ contribution approaches $\frac{3 N}{2}$, but there are multiple other states with the same energy, leading to degeneracy. For example, in the case of $N=2,|0,2,0\rangle$ has the same energy as $|1,0,1\rangle$, namely, 3 . Hence in contrast with the Lieb-Liniger model, these eigenstates remain the combination of several other states with an energy equal to $\frac{3 N}{2}$ even for vanishing interactions.

Even if the excited eigenstate did form a dark soliton, it would still be hard to realize this state in experiments. Therefore we decided to follow a different approach and try to replicate the experimental procedure of phase imprinting. This method creates a dark soliton in a BEC via a pulse of a far-detuned laser applied to one half of the condensate and so creates a phase difference between the left and the right sides. The length of this pulse is tuned to create a phase difference of $\pi$ and hence causes emergence of the dark soliton. There are not many papers discussing the phase-imprinting method in the many-body approach [41]. To the best of our knowledge, it has been applied only together with density engineering, which is not the case in the experimental realization. As in real life, our implementation of phase imprinting modifies only the phase of the wave function and is equivalent to multiplying the ground-state wave function by an arbitrary phase factor,

$$
\Psi\left(x_{1}, x_{2}, \ldots, x_{N}\right)=\Phi\left(x_{1}, x_{2}, \ldots, x_{N}\right) e^{i \phi\left(x_{1}, x_{2}, \ldots, x_{N}\right)},
$$

where $\Psi$ is the MB wave function of a solitonic state, $\Phi$ is the ground-state wave function, and $\phi$ is an arbitrary phase factor. For numerical convenience we choose $\phi\left(x_{1}, x_{2}, \ldots, x_{N}\right)=$ $\sum_{j}^{N} \tan ^{-1}\left(\alpha x_{j}\right)$, where $\alpha$ is the parameter changing the sharpness of the phase jump, which can also be controlled in experiments. The optimal sharpness of the phase jump has to be tuned to fit the healing length of the soliton. This means that the stronger the interaction, the narrower the phase jump has to be. It has been optimized by the trial-and-error method. In the case of a phase step that was too wide, the dip in density was indistinguishable from the background after less than a quarter of a period. A phase jump that is too narrow causes very high excitations and will demand a very large basis to obtain correct results. For now, the solitonic wave function $\Psi$ is merely an initial condition. We derive the time evolution of the system by expressing $\Psi$ in the basis of eigenstates of the system as

$$
\begin{gathered}
\Psi\left(x_{1}, x_{2}, \ldots, x_{N}, t\right)=\sum_{i} \beta_{i} \psi_{i}\left(x_{1}, \ldots, x_{N}\right) \exp \left(-i E_{i} t\right), \\
\beta_{i}=\int d x_{1} \ldots d x_{N} \psi_{i}^{*}\left(x_{1}, \ldots, x_{N}\right) \Psi\left(x_{1}, \ldots, x_{N}\right),
\end{gathered}
$$

where $\psi_{i}\left(x_{1}, \ldots, x_{N}\right)$ and $E_{i}$ are the eigenstates and the eigenvalues of Hamiltonian (4). In order to visualize a soliton, we derive the one-particle density:

$$
\begin{aligned}
\rho\left(x_{1}, t\right)= & \int d x_{2} \ldots d x_{N} \Psi^{*}\left(x_{1}, \ldots, x_{N}, t\right) \Psi\left(x_{1}, x_{2}, \ldots\right. \\
& \left., x_{N}, t\right) .
\end{aligned}
$$

As we aim to obtain the MF dark solitons from Eq. (2) and compare them with the MB solutions, we employ an analogous scheme. First, we find a ground state $\psi_{\mathrm{GPE}}(x)$ for given parameters by using the well-known imaginary time evolution technique. At this point, we can compare ground states obtained in the MF and MB approaches. Both density profiles and ground-state energies (up to a $2 \%$ difference for the highest $g$ ) are in very good agreement for both dipolar and 

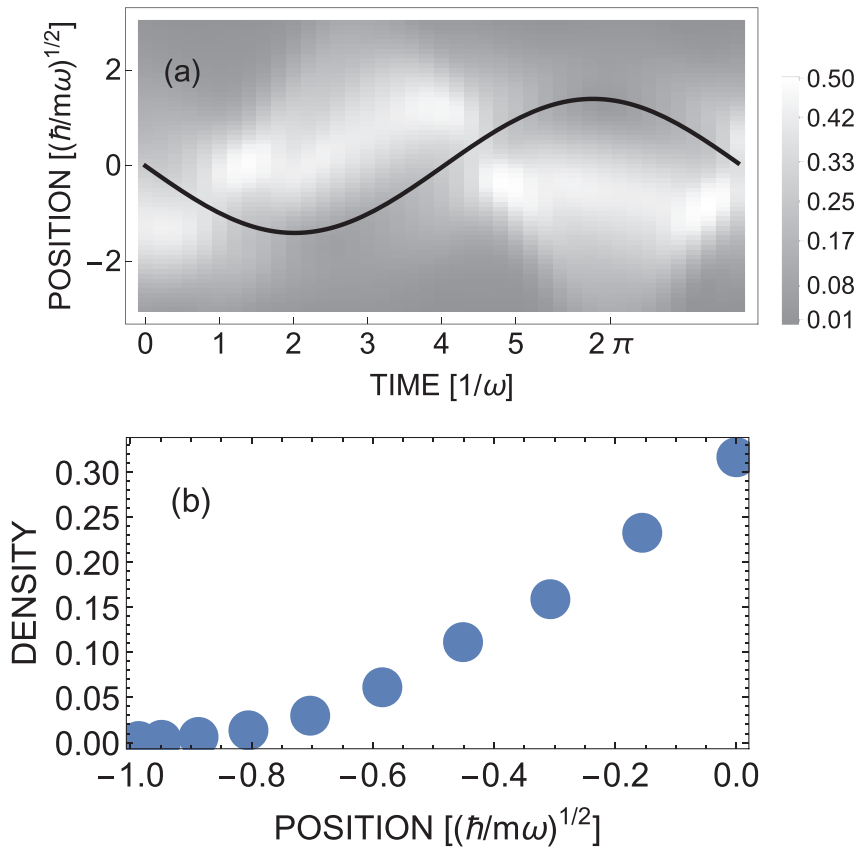

FIG. 2. (a) One-particle density evolution in time of a manybody dipolar dark soliton (solid black line) and a sloshing motion of the background (white trace) for $N=6$ particles and $g=0.9$. The dark soliton is moving at a frequency of $0.87 \omega$ and the cloud's sloshing motion oscillates with the frequency of the trap $\omega$. (b) The density in the dip plotted as a function of the position of the soliton. The depth of the soliton is dependent on its notch position: the soliton is shallow (gray) in the middle of the trap and becomes deep (black) at the turning point.

contact interactions and for all coupling strengths considered in this work. Next, we imprint the same phase as in the MB calculations, namely, $\Psi_{\mathrm{GPE}}(x)=\psi_{\mathrm{GPE}}(x) e^{i \phi_{\mathrm{MF}}(x)}$, with $\phi_{\mathrm{MF}}=$ $\tan ^{-1}(\alpha x)$. Finally, we evolve Eq. (2) in a standard real-time evolution with $\Psi_{\mathrm{GPE}}(x)$ as an initial condition.

Note that we can calculate the quantum depletion for any many-body state, in particular, for a many-body ground state before and after phase imprinting, by diagonalizing a single-particle density matrix constructed from the MB wave function. It provides a tool for comparing mean-field and many-body results.

\section{RESULTS}

Having a model of the experimental method of phase imprinting and being able to calculate the evolution of dark solitons, we can focus on the properties of contact and dipolar MB solitons and compare them with the MF results. First, we would like to focus our attention on general aspects of the evolution of many-body solitons in a harmonic trap. In order to study the evolution of the system we plot the oneparticle density as a function of time and space in Fig. 2 for $N=6$ dipolar bosons and $g=0.3$. This reveals that the phaseimprinting method causes not only the emergence of the dark soliton but also the motion of the whole cloud in the form of a density peak initially moving in the opposite direction to the soliton. As shown in the bottom panel in Fig. 2, the density in the middle of the soliton depends on its position. This
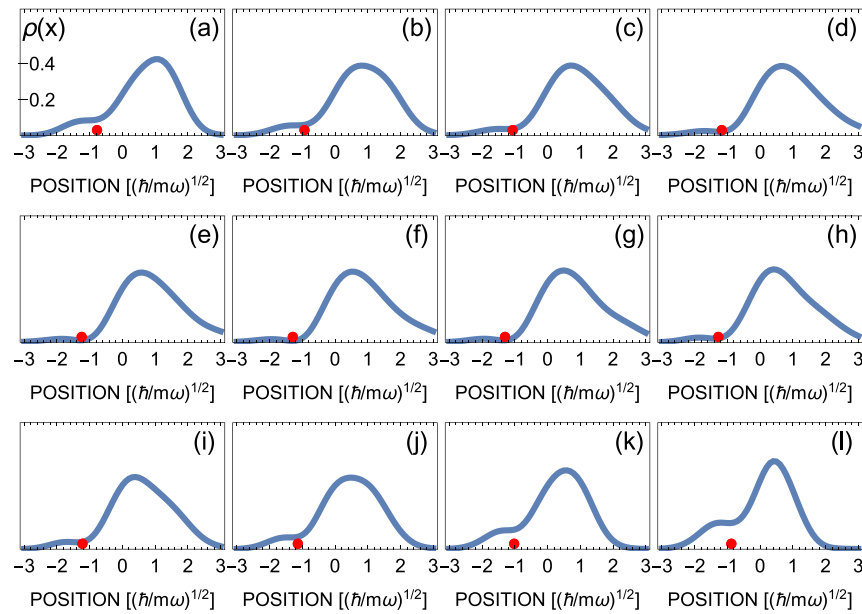

FIG. 3. (a)-(1) Sequence of images showing the spatial density profile in consecutive time steps for the situation in Fig. $2(N=6$ dipolar particles and $g=0.9$ ). The initial frame (upper-left corner) corresponds to time $t=0.2$ in units of $\frac{1}{\omega}$. The red dot indicates the position of the soliton. The soliton becomes deeper until the density reaches 0 , then the soliton bounces from the trap and begins moving to the other side of the trap. Once moving towards the center of the trap it becomes shallower.

effect of periodic, position-dependent transformation from a gray to a black soliton has been reported in [12]. Plots of density profiles in consecutive time steps shown in Fig. 3 reveal more details of soliton evolution. The local density minimum moves from the center of the trap towards the left side as long as the density of the notch is greater than 0 . When the soliton becomes black, namely, when the one-particle density in the dip reaches 0 , its velocity also equals 0 , both indicating a turning point. The soliton begins to move right and becomes shallow in the center of the trap. The relation between the depth of the dark soliton and its velocity is one of the fundamental properties of solitons and has been studied in a number of papers $[4,5,42]$.

As we have pointed out, the phase-imprinting method creates a soliton but also a sloshing motion of the cloud. An analogous effect has been observed in an experiment implementing phase imprinting [8]. The shock wave does not form ripples but rather a sloshing mode because of the small number of atoms. Both the shock wave and the soliton oscillate in the trap harmonically but the shock wave oscillates with the trap frequency. The difference between the frequencies causes dephasing of the center of mass and the soliton movement. It is thus worthwhile to study the frequency of the soliton movement, as it is one of the factors differentiating contact and dipolar solitons in the MF approach [15].

One of the properties of contact solitons revealed in a number of studies $[10,42,43]$ is that the frequency of oscillation does not depend on the strength of interactions and equals $\omega_{\mathrm{TF}}=\frac{1}{\sqrt{2}} \omega$. However, this result is obtained in the Thomas-Fermi limit assuming that the background density varies slowly on the scale of the soliton. The kinetic energy of particles in the TF limit can be neglected compared to the interaction energy. However, satisfying this condition in the case of small systems would require very strong interactions causing atoms to deplete the ground state and thus making our 


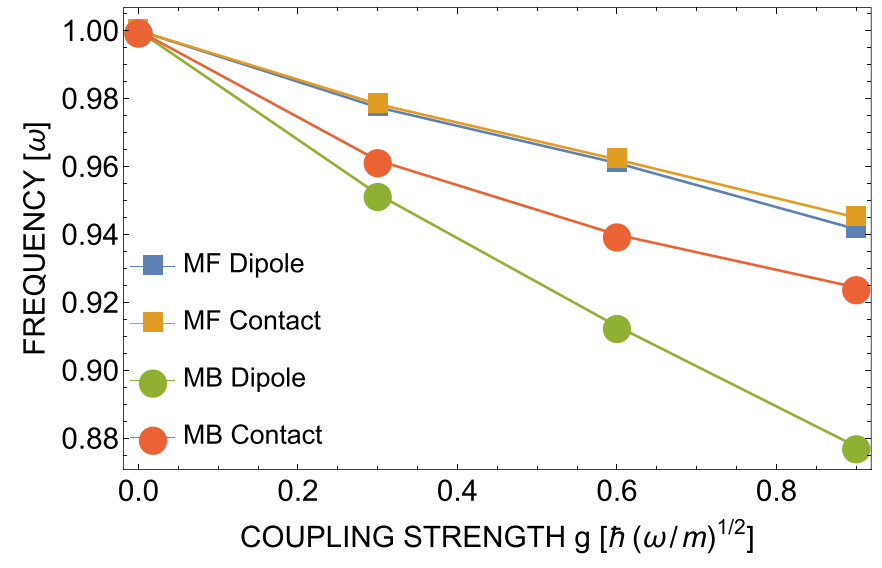

FIG. 4. Comparison of the frequency as a function of the coupling strength $g$ for contact and dipolar interactions in the many-body (circles) and mean-field (squares) approaches for $N=6$ particles. Mean-field solitons are almost indistinguishable from dipolar ones, being only slightly slower. On the other hand, many-body solitons differ significantly. Dipolar solitons always oscillate more slowly as for the MF dark solitons in the TF regime studied in [15]. Lines are guides for the eye.

and the mean-field results incomparable. Our studies focused on the case of small systems in the many-body approach far from the TF limit (even for the strongest interaction case considered here, the kinetic energy is $15 \%$ of the total energy) and with the quantum depletion of the ground state before phase imprinting not exceeding $5 \%$.

In order to analyze the frequency of oscillation, we trace the position of a local minimum of the one-particle density (in the MB approach) or condensate wave function (in the MF picture) in consecutive time steps. We trace the minimum until it crosses the center of the trap, which gives us half of the period.

A recent paper investigating dipolar solitons in the MF approach revealed significant differences between contact and dipolar solitons in the TF regime [15], one of them exhibited by the frequency of oscillations. In contrast to previously described mean-field contact solitons, the frequency of dipolar solitons depends on the interaction strength, and in general, the dipolar soliton frequency is lower than that obtained for contact solitons. It is thus worthwhile to ask whether many-body solitons exhibit similar behavior already for weak interactions.

To answer this question we directly compare many-body and mean-field solitons for contact and dipolar interactions. We plot the frequencies obtained for a system of $N=6$ particles for an increasing coupling strength $g$ in Fig. 4. First, we note that the frequencies of MF and MB solitons differ significantly. In the MF picture, contact and dipolar solitons are almost indistinguishable. On the contrary, MB dipolar solitons oscillate much more slowly than contact ones.

It is thus important to ask why the MF model predicts different results than the MB approach when applied to the studied system. The main factor contributing to the difference between the two methods is phase imprinting. Before imprinting, the systems are comparable, as the depletion of the MB ground state does not exceed 5\% (energies of the ground state and the MF solution do not differ by more than $5 \%$ also). After the procedure, it increases by $10 \%-20 \%$ depending on the sharpness of the phase jump. This means that the excited fraction can no longer be neglected in the MB calculations, while it is not present at all in the MF case. This is a very important observation, as the depletion of the ground state is fundamentally bound with the phaseimprinting method and needs to be taken into consideration when studying small systems both theoretically and experimentally. Growing quantum depletion after phase imprinting explains also the significant difference between dipolar and short-range solitons in the many-body picture and its absence in the MF case. The actual shape of the potential comes to play in the MB results, as more single-particle orbitals have to be involved to describe the ground-state properly. On the other hand, the MF wave function does not vary at the scale given by the range of dipolar interaction. Hence, for systems far from the TF regime, the dipolar interacting scenario differs very little from the short-range interaction case.

Having discussed the differences between MF and MB results, we focus on the properties of MB contact and dipolar solitons. The system proves to be interaction sensitive, as the frequency varies significantly with both the strength and the range of the interaction. In both cases, the frequency decreases with increasing coupling strength $g$. The dipolar solitons always oscillate more slowly than their short-range counterparts. As we are far from the TF limit, the frequency of contact solitons differs from $\frac{1}{\sqrt{2}} \omega$.

We have investigated not only the frequency of solitons' oscillations but also their lifetimes. While it is hard to define a sharp condition for the soliton to be indistinguishable from the background, we noted that contact solitons live significantly longer than their dipolar counterparts, with the lifetime strongly dependent on the coupling strength $g$.

\section{CONCLUSIONS}

The goal of this paper is to compare dark solitons in the mean-field and many-body approaches for contact and dipolar interactions. We have begun our many-body analysis by calculating the eigenstates and energies of the system via the numerical diagonalization of the Hamiltonian matrix.

In order to study many-body solitons in a harmonic trap we have introduced the multiatom version of the phaseimprinting method. Just as in the classic experiment it causes not only the soliton but also the sloshing motion to appear. These waves oscillate at different frequencies and thus movements of the soliton and the center of mass are decoupled. We investigate the frequency of oscillation of dark solitons to reveal similarities and differences between contact and dipolar solitons and compare our many-body analyses with the mean-field results.

The frequency of oscillations for dipolar solitons strongly depends on the coupling strength and is lower compared to that of contact solitons for the corresponding interaction strength. For comparison, we have also analyzed the contact and dipolar solitons in our small system induced by the phaseimprinting method at the MF level. The MF approach fails in the case of our system, as the dipolar and contact solitons are almost identical and their properties differ significantly 
from those of the MB solitons. The reason for the discrepancy is related to phase imprinting. The phase-imprinting method increases the quantum depletion of the ground state and the excited fraction is no longer negligible. Accordingly, a growing share of quickly oscillating excited quantum harmonic oscillator states influences the dynamics of the system within the MB description. Hence, the difference between contact and dipolar interactions is revealed also.

\section{ACKNOWLEDGMENTS}

We thank K. Pawłowski for his careful and critical reading of the manuscript and fruitful discussions. This work was supported by (Polish) National Science Center Grants No. 2016/21/N/ST2/03432 (R.O. and M.K.) and 2015/19/B/ST2/02820 (K.R.). The Center for Theoretical Physics is a member of KL FAMO.
[1] T. Dauxois and M. Peyrard, Physics of Solitons (Cambridge University Press, Cambridge, UK, 2006).

[2] D. Frantzeskakis, J. Phys. A: Math. Theor. 43, 213001 (2010).

[3] Y. S. Kivshar and B. Luther-Davies, Phys. Rep. 298, 81 (1998).

[4] V. E. Zakharov and A. B. Shabat, Zh. Eksp. Teor. Fiz. 61, 118 (1971).

[5] V. E. Zakharov and A. B. Shabat, Zh. Eksp. Teor. Fiz. 64, 1627 (1973) [Sov. Phys. JETP 37, 823 (1973)].

[6] T. Tsuzuki, J. Low Temp. Phys. 4, 441 (1971).

[7] A. Hasegawa and F. Tappert, Appl. Phys. Lett. 23, 171 (1973).

[8] S. Burger, K. Bongs, S. Dettmer, W. Ertmer, K. Sengstock, A. Sanpera, G. V. Shlyapnikov, and M. Lewenstein, Phys. Rev. Lett. 83, 5198 (1999).

[9] J. Denschlag, J. E. Simsarian, D. L. Feder, C. W. Clark, L. A. Collins, J. Cubizolles, L. Deng, E. W. Hagley, K. Helmerson, W. P. Reinhardt et al., Science 287, 97 (2000).

[10] T. Busch and J. R. Anglin, Phys. Rev. Lett. 84, 2298 (2000).

[11] A. Weller, J. P. Ronzheimer, C. Gross, J. Esteve, M. K. Oberthaler, D. J. Frantzeskakis, G. Theocharis, and P. G. Kevrekidis, Phys. Rev. Lett. 101, 130401 (2008).

[12] C. H. Tenorio, E. V. Vargas, V. N. Serkin, M. A. Granados, T. L. Belyaeva, R. P. Moreno, and L. M. Lara, Quantum Electron. 35, 929 (2005).

[13] V. N. Serkin, Optik 173, 1 (2018).

[14] V. N. Serkin, A. Hasegawa, and T. L. Belyaeva, Phys. Rev. Lett. 98, 074102 (2007).

[15] T. Bland, K. Pawłowski, M. J. Edmonds, K. Rzążewski, and N. G. Parker, Phys. Rev. A 95, 063622 (2017).

[16] K. Pawłowski and K. Rzążewski, New J. Phys. 17, 105006 (2015).

[17] T. Bland, M. J. Edmonds, N. P. Proukakis, A. M. Martin, D. H. J. O’Dell, and N. G. Parker, Phys. Rev. A 92, 063601 (2015).

[18] M. Lu, N. Q. Burdick, S. H. Youn, and B. L. Lev, Phys. Rev. Lett. 107, 190401 (2011).

[19] T. Maier, H. Kadau, M. Schmitt, A. Griesmaier, and T. Pfau, Opt. Lett. 39, 3138 (2014).

[20] K. Aikawa, A. Frisch, M. Mark, S. Baier, A. Rietzler, R. Grimm, and F. Ferlaino, Phys. Rev. Lett. 108, 210401 (2012).

[21] E. H. Lieb and W. Liniger, Phys. Rev. 130, 1605 (1963).

[22] E. H. Lieb, Phys. Rev. 130, 1616 (1963).
[23] P. P. Kulish, S. V. Manakov, and L. D. Faddeev, Theor. Math. Phys. 28, 615 (1976).

[24] M. Ishikawa and H. Takayama, J. Phys. Soc. Jpn. 49, 1242 (1980).

[25] R. Kanamoto, L. D. Carr, and M. Ueda, Phys. Rev. Lett. 100, 060401 (2008).

[26] A. D. Martin and J. Ruostekoski, Phys. Rev. Lett. 104, 194102 (2010).

[27] O. Fialko, M.-C. Delattre, J. Brand, and A. R. Kolovsky, Phys. Rev. Lett. 108, 250402 (2012).

[28] J. Sato, R. Kanamoto, E. Kaminishi, and T. Deguchi, Phys. Rev. Lett. 108, 110401 (2012).

[29] A. Syrwid and K. Sacha, Phys. Rev. A 92, 032110 (2015).

[30] A. Syrwid, M. Brewczyk, M. Gajda, and K. Sacha, Phys. Rev. A 94, 023623 (2016).

[31] G. C. Katsimiga, S. I. Mistakidis, G. M. Koutentakis, P. G. Kevrekidis, and P. Schmelcher, New J. Phys. 19, 123012 (2017).

[32] S. S. Shamailov and J. Brand, Phys. Rev. A 99, 043632 (2019).

[33] E. Kaminishi, T. Mori, and S. Miyashita, J. Phys. B: At., Mol. Opt. Phys. 53, 095302 (2020).

[34] M. Greiner, O. Mandel, T. Esslinger, T. W. Hänsch, and I. Bloch, Nature 415, 39 (2002).

[35] F. Serwane, G. Zürn, T. Lompe, T. Ottenstein, A. Wenz, and S. Jochim, Science 332, 336 (2011).

[36] F. Meinert, M. Panfil, M. J. Mark, K. Lauber, J.-S. Caux, and H.-C. Nägerl, Phys. Rev. Lett. 115, 085301 (2015).

[37] S. Baier, M. J. Mark, D. Petter, K. Aikawa, L. Chomaz, Z. Cai, M. Baranov, P. Zoller, and F. Ferlaino, Science 352, 201 (2016).

[38] S. Baier, D. Petter, J. H. Becher, A. Patscheider, G. Natale, L. Chomaz, M. J. Mark, and F. Ferlaino, Phys. Rev. Lett. 121, 093602 (2018).

[39] M. Płodzień, D. Wiater, A. Chrostowski, and T. Sowiński, arXiv:1803.08387.

[40] R. Ołdziejewski, W. Górecki, K. Pawłowski, and K. Rzążewski, Phys. Rev. A 97, 063617 (2018).

[41] S. Krönke and P. Schmelcher, Phys. Rev. A 91, 053614 (2015).

[42] N. G. Parker, N. P. Proukakis, and C. S. Adams, Phys. Rev. A 81, 033606 (2010).

[43] P. O. Fedichev, A. E. Muryshev, and G. V. Shlyapnikov, Phys. Rev. A 60, 3220 (1999). 